\title{
Wie Unternehmen und Konsumenten voneinander lernen können
}

\author{
Umweltschonende Konsum- und Produktions- \\ muster sind ein Kernpunkt einer nachhaltigen \\ Entwicklung. Durch partizipative Produktent- \\ wicklung entstehen Lerneffekte, mit denen \\ Verbraucher und Produzenten dieser Heraus- \\ forderung besser gerecht werden können. \\ Von Marlen Arnold, Bernd Siebenhüner \\ und Lenz Hoser
}

D er Dialog von Kunden und Unternehmen hilft diesem Land. Ich finde ihn notwendig, weil es letztendlich mein Geld ist, wenn ich etwas kaufe. Deshalb möchte ich auch mehr Mitsprache haben - so die Worte eines Konsumenten, der aktiv im INNOCOPE-Verfahren (INNOvation through COnsumer-integrated Product dEvelopment) mitgewirkt hat. Dieses im Rahmen des GELENA-Projekts konzipierte Produktentwicklungsverfahren zielt auf die Initiierung wechselseitiger Lernprozesse zwischen Konsument(inn)en und Unternehmensvertreter(inne)n. In dem vom Bundesministerium für Bildung und Forschung geförderten Vorhaben sollte ein Produkt entwickelt werden, das einen aktiven Beitrag zum Klimaschutz leisten kann. Durchgeführt wurde das Verfahren mit dem Unternehmen HAWK Bikes E\&M GmbH im Jahr 2005 zusammen mit Wissenschaftler(inne)n der Universität Oldenburg und des Instituts für ökologische Wirtschaftsforschung (1). Wie die Erfahrungen zeigen, können über derartige Verfahren sowohl wechselseitige Lernprozesse zwischen Unternehmen und Konsument(inn)en initiiert als auch konkrete, marktfähige und klimaschonende Produkte entwickelt werden.

\section{Partizipative Produktentwicklung}

Laut Agenda 21 stellen nicht nachhaltige Verbrauchs- und Produktionsmuster die Hauptursache für die allmähliche Zerstörung der globalen Umwelt dar (BMU 1992). Im Sinne einer geteilten Verantwortung für Nachhaltigkeit kommt sowohl Unternehmen als auch den Verbraucher(inne)n Verantwortung zu. Während die Konsument(inn)en diese derzeit vor allem über ihre Kaufentscheidung ausüben können, ist ihre direkte Einflussnahme auf die Produktgestaltung als zentralem unternehmerischem Entscheidungsbereich bisher gering. Nutzer(innen) stellen zwar eine wesentliche Stakeholdergruppe der Unternehmen dar, ihre Interessen werden aber meist nur mittelbar über
Marketing und Marktforschung in die Produktentwicklung einbezogen. Gleichwohl wird in der jüngeren Innovationsforschung die Bedeutung von Kund(inn)en in Innovationsprozessen hervorgehoben (Wecht 2006; Hippel 1998). Die Entwicklung eines partizipativen Produktentwicklungsverfahrens im Rahmen des GELENA-Projekts knüpft hier an und bezieht darüber hinaus Erkenntnisse aus der Partizipationsforschung, der Technik- und Innovationsbewertung, der Nachhaltigkeitsforschung sowie der Wissensgenerierung ein (Joss 2002; Siebenhüner 2004). Im Rahmen einer partizipativ angelegten Entwicklung klimaschonender Produkte können nicht nur die spezifischen Wünsche und Bedürfnisse der Konsument(inn)en unmittelbarer berücksichtigt, sondern auch ihr Handlungs- und Alltagswissen aktiviert und integriert werden. Durch die frühzeitige Beteiligung von Nutzer(inne)n ergibt sich insbesondere die Möglichkeit, Anwendungs- und Kontextwissen einzubeziehen und so zusätzliche ökologische und soziale Potenziale in der Nutzung von Produkten zu erschließen.

\section{Konzept partizipativer Produktentwicklung im Klimaschutz}

Unter Einbeziehung von sowohl Nutzer(inne)n als auch Unternehmen sollte im Rahmen von GELENA mithilfe von geeigneten Partizipationsmethoden ein Verfahren zur Entwicklung von klimafreundlichen Produkten entworfen werden. Hierbei werden insbesondere die folgenden Ziele verfolgt:

- Initiierung und Unterstützung von wechselseitigen Lernprozessen zur Produktentwicklung und zum Klimaschutz,

- gleichberechtigte Einbeziehung verschiedener Wissenstypen (Alltagswissen, Expertenwissen),

I Empowerment der beteiligten Konsument(inn)en,

- Entwicklung neuer oder verbesserter Produkte im Hinblick auf den Klimaschutz.

In Vorbereitung auf das Verfahren wurden mehrere Arbeitsschritte durchlaufen. Hierzu zählt die Aufbereitung von Bedürfnisfeldstudien (Beschorner 2005) und Unternehmensfallstudien (Siebenhüner 2006). Auf Basis dieser Sondierungsstudien wurden geeignete Produkte identifiziert. Daneben konnten wesentliche Fragen zu Trendentwicklungen und zu Nachhaltigkeit und Klimaschutz im jeweiligen Bedarfsfeld erhellt werden. Die Auswertung der Einzelfallstudien bot für die Frage der Verfahrensentwicklung einige Anhaltspunkte. So zeigte sich, dass die Kommunikation zwischen der Umwelt- beziehungsweise Nachhaltigkeitsabteilung und der Produktentwicklung häufig $\rightarrow$ 
nicht gut entwickelt ist und daher Unternehmensvertreter(innen) an relevanten Schnittstellen und mit unterschiedlichen Tätigkeitsschwerpunkten gemeinsam am Verfahren beteiligt sein sollten. Parallel dazu ist eine Modellierungssoftware entwickelt worden, die im GELENA-Produktentwicklungsverfahren zur Anwendung kam (2). Die Modellierungssoftware (EcoClass, Ecological and Climate Assessment of Product Innovations), ökologische und Klimabewertung von Produktinnovationen, ermöglicht, die ökologische Relevanz des zu entwickelnden Produkts und verschiedener Nutzungsweisen abzubilden und zu untersuchen.

\section{Durchführung}

Für das produktbezogene Partizipationsverfahren konnte eine erfolgreiche Kooperation mit HAWK Bikes, einem Unternehmen im Produktbereich Fahrräder beziehungsweise Elektrofahrräder aufgebaut werden (3). Die Anwendung des INNOCOPE-Verfahrens bot sich am Beispiel des nachhaltigen Produktes Pedelecs (Pedal electric cycle) aus unterschiedlichen Gründen an. Pedelecs ermöglichen als Fahrräder mit elektrischer Tretunterstützung eine höhere Geschwindigkeit und größere Reichweite als konventionelle Fahrräder, der benötigte Strom kann mittels regenerativer Energien bezogen werden und Pedelecs können daher insbesondere in der Stadt als attraktive und zugleich umwelt- und klimafreundlichere Alternative zu Autos, Motorrädern oder Motorrollern betrachtet werden. Dies zeigte auch die im Workshop eingesetzte Computersimulation EcoClass zur Darstellung der Umweltauswirkungen unterschiedlicher Verkehrsträger.

Das INNOCOPE-Verfahren gliedert sich in eine Vorbereitungsphase und eine etwa halbjährige Workshopphase, wobei die konkrete Umsetzung und Detailplanung in enger Abstimmung mit HAWK Bikes erfolgte. In der Vorbereitungsphase ging es zunächst um die Abstimmung gemeinsamer Ziele und Erwartungen mit dem Unternehmen. Die anschließende Ansprache und Gewinnung der Teilnehmer(innen), die sich etwa je zur Hälfte aus Fahrradnutzer(inne)n und Personen, die bislang keine oder kaum Fahrräder nutzen, zusammensetzten, wurde ebenfalls mit dem Unternehmen abgestimmt. An den drei Workshops, die am Unternehmensstandort Berlin durchgeführt wurden, haben dieselben Konsument(inn)en teilgenommen. Von Unternehmensseite waren der Geschäftsführer, der Produktmanager, der Designer sowie teilweise ein Vertriebsmitarbeiter anwesend. Im ersten Workshop wurde ein Schwerpunkt auf die Vermittlung von Klimaschutzwissen gelegt. Da im Mittelpunkt des INNOCOPE-Verfahrens eine ökologisch ausgerichtete Produktentwicklung steht, diente der Vortrag zum Klimawandel auch dazu, die partizipative Produktentwicklung entsprechend vorzubereiten. In den drei aufeinander aufbauenden Workshops haben die Unternehmensvertreter von HAWK Bikes und Craftsmen Design gemeinsam mit dem GELENA-Team und rund 20 Berliner Konsument(inn)en das Produkt Pedelec weiterentwickelt. In den drei Workshops wurden

Anforderungen an Pedelecs diskutiert, Ideen für die Verbesserung von Pedelecs entwickelt und bewertet sowie Marketingmaßnahmen für die bessere Bekanntmachung und den Vertrieb von Pedelecs erarbeitet.

In den Workshops kamen jeweils verschiedene Methoden wie Präsentation, Plenumsdiskussion, Kleingruppenarbeit, Metaplan und Brainstorming zum Einsatz. Dabei wurden zum einen Alltagssituationen simuliert (Probefahrt mit Pedelecs, Bildmappen, provokante Fragen) und zum anderen Kreativitätstechniken (Assoziationstechniken, Analogiemethoden und Bewertungsmethoden) eingesetzt, die mögliche Verständigungsschwierigkeiten zwischen Unternehmensvertretern und Nutzern ausgleichen beziehungsweise vermindern sollen.

$\mathrm{Zu}$ dem Verfahren wurde eine Begleitforschung durchgeführt, die dazu diente, das im Projekt entwickelte Verfahren zu bewerten. Hierbei ging es vor allem darum zu prüfen, inwieweit die gesetzten Ziele (Lernen, Empowerment, ökologische Innovationen) erreicht wurden. Gleichzeitig sollte die Praktikabilität des Verfahrens geprüft und Empfehlungen zur Durchführung partizipativer Verfahren gegeben werden. Hierzu wurden die durchgeführten Workshops dokumentiert und ausgewertet, zudem wurden Interviews mit den beteiligten Konsument(inn)en und Unternehmensvertretern geführt. Dabei wurden die Unternehmensvertreter unter anderem zu folgenden Themengruppen befragt: Erwartungen an und Bewertung von INNOCOPE, Konsument(inn)enbedürfnisse und Produktentwicklung, Klimaschutz und Klimawandel.

\section{Erfolge gemeinsamer Produktentwicklung}

Ende September 2005 stellte HAWK Bikes im dritten Workshop seinen ersten Pedelec-Prototypen vor. Nicht nur hinsichtlich des Gewichts von $20 \mathrm{~kg}$, der Reichweite von $30 \mathrm{~km}$ und der tretunterstützten Höchstgeschwindigkeit von $25 \mathrm{~km} / \mathrm{h}$, sondern auch bezüglich des Designs von Rahmen, Dynamo und Beleuchtung hatte HAWK Bikes die Anforderungen der Konsument(inn)en umgesetzt. Nach weiteren Überarbeitungen läuft die Vermarktung des Pedelecs Swizzbee 25L seit Sommer 2006. Die beteiligten Konsument(inn)en waren von der gemeinsamen Arbeit in den Workshops und dem Ergebnis begeistert: „Es ist ein vielseitiges, richtig schickes Pedelec herausgekommen, das auch meinen Designanforderungen gerecht wird. Das Unternehmen hat sich wirklich bemüht, das Pedelec nahe an unse-

Abbildung 1: Vorstellung des PedelecPrototypen durch HAWK Bikes im dritten Workshop

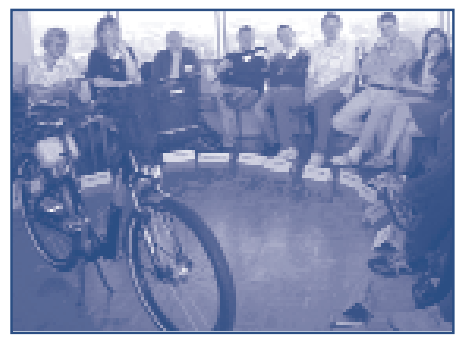
ren Ideen zu gestalten." (4). Auch die Unternehmensvertreter stellten wesentliche Merkmale des INNOCOPE-Verfahrens positiv heraus: „Gut war, dass wir direkt mit dem Endverbraucher in Kontakt getreten sind und erfahren konnten, was wirklich deren Anforde- 
rungen sind: So haben wir vor allem im Bereich Damenfahrräder sehr viele neue Informationen bekommen."

Insgesamt lässt sich erkennen, dass sich die Unternehmensvertreter im Laufe des Verfahrens zunehmend aufgeschlossener gegenüber neuen Erkenntnissen und Impulsen von Konsument(inn)en zeigen. Besonders relevant sind der direkte Kontakt und die Informationsweitergabe der Konsument(inn)en an die Unternehmensvertreter. Da der Fachhandel oftmals Informationen filtert und auf bereits existierende Produkte bezogen weitergibt, konnten die Unternehmensvertreter im Verfahren direkt Erfahrungswissen, zukunftsorientierte Informationen und Bedarfe von potenziellen Kund(inn)en erhalten. Bisher hatte das Unternehmen vor allem Einblicke in die Anforderungen von Spezialkund(inn)en wie Extremsportler(innen). Die in den Workshops anwesende Kund(inn)engruppe lieferte daher wichtige Erkenntnisse bezüglich der Anforderungen an die Alltagstauglichkeit von Pedelecs und Fahrrädern. Hinsichtlich der Kundenbedürfnisse und insbesondere der Anforderungen an Damenräder haben alle Unternehmensvertreter einen Wissenszuwachs anerkannt. Die Einblicke in die Nutzungsweisen und Bedarfe von Konsument(inn)en haben zum Lernen aufseiten des Unternehmens geführt. Die Kundenanforderungen sollen aktiv aufgenommen und in zukünftige Produktentwicklungen integriert werden. Es wurde auch ein Bedarf an weiterführenden Marktforschungsaktivitäten, wie zum Beispiel repräsentativen Produktbefragungen, erkannt.

Was den Wissensstand der Unternehmensvertreter $\mathrm{zu}$ Klimaaspekten betrifft, konnten hingegen nur geringe Veränderungen festgestellt werden. Obwohl viele Aspekte nach wie vor unterbelichtet blieben, wie zum Beispiel unternehmerische Handlungsoptionen zum Klimaschutz und konkretes Wissen sowie Handlungswissen zu Klima- und Umweltschutz, wurde der Klimawandel anerkannt. Nach dem Verfahren wiesen die Unternehmensvertreter mehr Wissen zu Klimawandel und Klimaschutz auf. Zudem erkannten die Unternehmensvertreter verstärkt klimarelevante Zusammenhänge ihrer eignen Produkte und Produktionsprozesse. So hat das Bewusstsein für die ökologische Relevanz der Lebenszyklusphasen (Rohstoffbedarf und Nutzung) des Pedelecs, der Rohstoffauswahl sowie des Energiebedarfs zugenommen. Der Beitrag zum Klimaschutz mit Pedelec als Substitut für den motorisierten Stadtverkehr wird derzeit vom Unternehmen aktiv in der Internetpräsenz beworben.

\section{Fazit}

Insgesamt hat sich HAWK Bikes im Prozessverlauf das zunutze gemacht, was in der neueren Innovationsforschung gefordert wird. Die Einbindung des kreativen Potenzials und des Alltagswissens von Konsument(inn)en und Nutzer(inne)n in die Entwicklung von Produktinnovationen. Gerade kleine und mittelständische Unternehmen, die aufgrund finanzieller Engpässe nicht auf systematische Methoden zur Ermittlung von Kundenwünschen zurückgreifen können, profitieren so von Partizipationsverfahren. Die Zusammenarbeit mit Endkonsu- ment(inn)en ist für sie „auf jeden Fall eine Lehre“. Zu den Erfolgfaktoren im vorliegenden Fall zählt zum einen die aktive Unterstützung durch die Unternehmensleitung. Zum anderen ist HAWK Bikes ein flexibles und hochgradig entwicklungs- und innovationsorientiertes Unternehmen, das auch methodischen Neuerungen gegenüber aufgeschlossen ist. Schließlich scheint auch die Auswahl des Produktes fruchtbar für den Verlauf des Verfahrens gewesen zu sein, da es hinreichend anschaulich, alltagsrelevant und für Konsument(inn)en von Interesse war. Für sich zogen die Unternehmensvertreter eine überaus positive Bilanz: „Der Vorteil derartiger Konsumentenworkshops ist die Weiterbildung von uns Herstellern. Als Unternehmer hat man eine bestimmte Idee von bestimmten Bereichen. Das INNOCOPE-Verfahren bringt einen weiter, um noch effizienter zu sein mit Produktentwicklung und Innovation. Ich fand es sehr gut.“

\section{Anmerkungen}

(1) www.gelena.net

(2) Siehe den Beitrag von Barth in diesem Heft

(3) www.hawkbikes.com

(4) Siehe den Beitrag von Hoffmann/Konrad in diesem Heft

\section{Literatur}

Beschorner, T. / Behrens, T. / Hoffmann, E. / Lindenthal, A. / Hage, M. / Thierfelder, B. / Siebenhüner, B.: Institutionalisierung von Nachhaltigkeit. Eine vergleichende Untersuchung der organisationalen Bedürfnisfelder Bauen \& Wohnen, Mobilität und Information \& Kommunikation. Marburg 2005.

BMU, Bundesministerium für Umwelt, Naturschutz und Reaktorsicherheit, (Hrsg.): Umweltpolitik : Konferenz der Vereinten Nationen für Umwelt und Entwicklung im Juni 1992 in Rio. Bonn 1992.

Hippel, E. von: The Dominant Role of Users in the Scientific Instrument Innovation Process. Research Policy. 5/1976. S.212-239.

Joss, S. / Bellucci, S. (Hrsg.): Participatory Technology Assessment. European Perspectives. London 2002.

Siebenhüner, B.: Social Learning and Sustainability Science: Which role can stakeholder participation play? International Journal of Sustainable Development 7, 2/2004. S.146-163.

Siebenhüner, B. / Arnold, M. / Hoffmann E. / Behrens, T. / Heerwart, S. / Beschorner T.: Organisationales Lernen und Nachhaltigkeit. Prozesse, Auswirkungen und Einflussfaktoren in sechs Unternehmensfallstudien. Marburg 2006.

Wecht, Ch.: Das Management aktiver Kundenintegration in der Frühphase des Innovationsprozesses, Wiesbaden 2006.

\section{AUTOREN + KONTAKT}

Marlen Arnold ist wissenschaftliche Mitarbeiterin an der Carl-von-Ossietzky Universität Oldenburg und im Forschungsfeld Ökologische Unternehmenspolitik am IÖW.

Carl-von-Ossietzky Universität Oldenburg, GELENA, 26111 Oldenburg. Tel.: 0441/798-4384, E-Mail: marlen.arnold@uni-oldenburg.de

Prof. Dr. Bernd Siebenhüner ist Juniorprofessor für Ökologische Ökonomie an der Carl-von-Ossietzky Universität Oldenburg, Leiter der Nachwuchsforschungsgruppe Gesellschafliches Lernen und Nachhaltigkeit (GELENA). Carl-von-Ossietzky Universität Oldenburg, 26111 Oldenburg. Tel.: 0441/798-4366, E-Mail: bernd.siebenhuener@uni-oldenburg.de Lenz Hoser ist Geschäftsführer von HAWK Bikes E\&M GmbH.
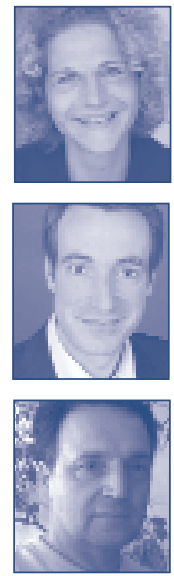

Tel.: 030/447221-11, E-Mail: lenz.hoser@hawkbikes.com 
(c) 20I0 Authors; licensee IÖW and oekom verlag. This is an article distributed under the terms of the Creative Commons Attribution Non-Commercial No Derivates License (http://creativecommons.org/licenses/by-nc-nd/3.o/), which permits unrestricted use, distribution, and reproduction in any medium, provided the original work is properly cited. 